Report no. 95/04

\title{
Stability Analysis of Galerkin/Runge-Kutta Navier-Stokes Discretisations on Unstructured Grids
}

\author{
M. B. Giles \\ Oxford University Computing Laboratory \\ Numerical Analysis Group
}

This paper presents a timestep stability analysis for a class of discretisations applied to the linearised form of the Navier-Stokes equations on a 3D domain with periodic boundary conditions. Using a suitable definition of the 'perturbation energy' it is shown that the energy is monotonically decreasing for both the original p.d.e. and the semi-discrete system of o.d.e.'s arising from a Galerkin discretisation on a tetrahedral grid. Using recent theoretical results concerning algebraic and generalised stability, sufficient stability limits are obtained for both global and local timesteps for fully discrete algorithms using Runge-Kutta time integration.

Subject classifications: AMS(MOS): 65M10, 65M20, 65M60, 76-08, 76N10

Key words and phrases: Navier-Stokes, method of lines, stability analysis

This work was supported by Rolls-Royce plc, DTI and EPSRC.

Oxford University Computing Laboratory

Numerical Analysis Group

Wolfson Building

Parks Road

Oxford, England OX1 3QD

E-mail: giles@comlab.oxford.ac.uk

April, 1997 


\section{Introduction}

One motivation for the analysis in this paper was the observation by Wigton of instabilities in Navier-Stokes calculations on structured grids [1]. It appeared that the instabilities might be connected to large variations in the level of turbulent viscosity arising quite properly in certain physical situations. A possible cause of the instability was thought to be the timestep definition which was based on Fourier stability theory assuming constant coefficients. Therefore, an objective of this analysis was to determine sufficient conditions for the stability of discretisations of the Navier-Stokes equations with nonuniform viscosity.

The second motivation was the requirement for timestep stability limits for viscous calculations on unstructured grids. Inviscid calculations are now being performed almost routinely on unstructured grids for complete aircraft geometries (e.g. $[2,3,4,5])$. Using energy analysis methods, Giles developed sufficient global and local timestep stability limits for a Galerkin discretisation of the Euler equations on a tetrahedral grid with two particular Runge-Kutta time integration schemes [6]; this has been used on an ad hoc basis for calculations using other algorithms including various upwinding and numerical smoothing formulations $[3,5]$. Through parallel computing and efficient multigrid algorithms for unstructured grids [5], there is now the computational power to perform extremely large Navier-Stokes calculations on unstructured grids, and so there is a need for the supporting numerical analysis to give accurate global and local timestep stability limits.

Fourier stability analysis can only be applied to linear finite difference equations with constant coefficients on structured grids, and so it is not appropriate for this application. There are two other well-documented stability analysis methods which can be used with linear discretisations with variable coefficients on unstructured grids. One is the energy method [7] which relies on the careful construction of a suitably defined 'energy' which can be proven to monotonically decrease. The difficulty is usually in constructing an appropriate definition for the energy, but when this method can be applied it is very powerful in giving a very strong form of stability. It is used in this paper to prove the stability of the original linearised form of the Navier-Stokes partial differential equations, and the semi-discretised system of coupled o.d.e.'s that is produced by the Galerkin spatial discretisation.

The other stability analysis technique involves consideration of the eigenvalues of the matrix representing the discretisation of the spatial differential operator. This leads to sufficient conditions for asymptotic stability, as $t \rightarrow \infty$ for unsteady calculations or as $n \rightarrow \infty$ for calculations using local timesteps. Unfortunately, there are well-documented examples such as the first order upwinding of the convection equation on a finite $1 \mathrm{D}$ domain (e.g. $[8,9,10]$ ) for which this is not a practical stability criterion because it allows an unacceptably large transient growth before the eventual exponential decay. The next section 
reviews this theory showing that the problem of large transient growth can arise whenever the spatial discretisation matrix is non-normal. It then presents recent results on algebraic and generalised stability for such applications giving sufficient conditions for stability. It is these new stability conditions which are used to construct sufficient stability limits for the full Galerkin/Runge-Kutta Navier-Stokes discretisation.

The analysis is performed for linear perturbations to a steady flow in which all flow variables are uniform with the exception of the three viscosity coefficients, $\mu$, the shear viscosity, $\lambda$, the second coefficient of viscosity, and $k$, the thermal diffusivity. This choice of model problem is critical in several ways. Although it is the linearisation of the laminar Navier-Stokes equations that is used, the viscosity coefficients can each be interpreted as the sum of the laminar value plus a turbulent value arising from some turbulence model. Accordingly, there is no assumption of any fixed relationship between the three quantities, either the Stokes hypothesis linking $\mu$ and $\lambda$, or the assumption of a constant Prandtl number linking $\mu$ and $k$. The uniformity of the other flow variables is essential for key parts of the analysis. However, a more fundamental aspect of the uniformity is that it gives a physical situation in which flow perturbations are naturally damped, and so the flow is stable. Therefore, an instability of the semi-discrete or fully discrete equations can be viewed justifiably as an incorrect behaviour. The timestep limit which gives the onset of this instability can then be defined as the maximum stable timestep. In contrast, if a vortex sheet were taken as the steady flow and then linear perturbations were analysed, it would be determined that both the analytic and discrete equations were unstable. Even worse, the timescale of the most unstable discrete mode would be proportional to $\Delta x$ so that it would be impossible to distinguish between a 'numerical instability' and the natural Helmholtz instability of the vortex sheet. It would not therefore be possible to use this alternative model problem to make any deductions about stable timestep limits.

After the following section reviewing numerical stability theory, there are separate sections for the analysis of the differential, semi-discrete and fully discrete Navier-Stokes equations. To focus attention on the important features of the stability analysis, many of the supporting details are presented in the three appendices.

\section{Review of stability theory for Runge-Kutta methods}

Discretisation of the scalar o.d.e.

$$
\frac{d u}{d t}=\lambda u
$$


using an explicit Runge-Kutta method with timestep $k$ yields a difference equation of the form

$$
u^{(n+1)}=L(\lambda k) u^{(n)}
$$

where $L(z)$ is a polynomial function of degree $p$

$$
L(z)=\sum_{m=0}^{p} a_{m} z^{m}
$$

with $a_{0}=a_{1}=1, a_{p} \neq 0$. Discrete solutions of this difference equation on a finite time interval $0 \leq t \leq t_{0}$ will converge to the analytic solution as $k \rightarrow 0$. In addition, the discretisation is said to be absolutely stable for a particular value of $k$ if it does not allow exponentially growing solutions as $t \rightarrow \infty$; this is satisfied provided $\lambda k$ lies within the stability region $S$ in the complex plane defined by

$$
S=\{z:|L(z)| \leq 1\}
$$

Examples of stability regions for different polynomials are given in Appendix A.

Suppose now that a real square matrix $C$ has a complete set of eigenvectors and can thus be diagonalised,

$$
C=T \Lambda T^{-1}
$$

with $\Lambda$ being the diagonal matrix of eigenvalues of $C$, and the columns of $T$ being the associated eigenvectors. The Runge-Kutta discretisation of the coupled system of o.d.e.'s,

$$
\frac{d U}{d t}=C U
$$

can be written as

$$
U^{(n+1)}=L(k C) U^{(n)}=T L(k \Lambda) T^{-1} U^{(n)},
$$

since

$$
C^{m}=\left(T \Lambda T^{-1}\right)^{m}=T \Lambda^{m} T^{-1}
$$

Hence

$$
U^{(n)}=T(L(k \Lambda))^{n} T^{-1} U^{(0)} .
$$

The necessary and sufficient condition for absolute stability as $n \rightarrow \infty$, requiring that there are no discrete solutions which grow exponentially with $n$, is therefore that $|L(k \lambda)| \leq 1$, or equivalently $k \lambda$ lies in $S$, for all eigenvalues $\lambda$ of $C$. If this condition is satisfied, then using $L_{2}$ vector and matrix norms it follows that

$$
\left\|U^{(n)}\right\| \leq\|T\|\|L(k \Lambda)\|^{n}\left\|T^{-1}\right\|\left\|U^{(0)}\right\| \leq \kappa(T)\left\|U^{(0)}\right\|,
$$

where $\kappa(T)$ is the condition number of the eigenvector matrix $T$. 
If the matrix $C$ is normal, meaning that it has an orthogonal set of eigenvectors then the eigenvectors can be normalised so that $\kappa(T)=1$. In this case, $\left\|U^{(n)}\right\|$ is a non-increasing function of $n$ and $\left\|U^{(n)}\right\|^{2}$ represents a non-increasing 'energy' which could be used in an energy stability analysis.

If $C$ is not normal, then the growth in $\left\|U^{(n)}\right\|$ is bounded by the condition number of the eigenvector matrix, $\kappa(T)$. Unfortunately, this can be very large indeed, allowing a very large transient growth in the solution even when for each eigenvalue $k \lambda$ lies strictly inside the stability region $S$ and so $\left\|U^{(n)}\right\|$ must eventually decay exponentially. This problem can be particularly acute when the matrix $C$ comes from the spatial discretisation of a p.d.e. in which case there is then a family of discretisations arising from a sequence of computational grids of decreasing mesh spacing $h$. It is possible in such circumstances for the sequence of condition numbers $\kappa(T)$ to grow exponentially, with an exponent inversely proportional to the mesh spacing [8]. There are two practical consequences of this exponential growth. In applications concerned with the behaviour of the solution as $t \rightarrow \infty$, it produces an unacceptably large amplification of machine rounding errors in linear computations and complete failure of the discrete computation in nonlinear cases. In applications concerned with a finite time interval, $0 \leq t \leq t_{0}$, it prevents convergence of the discrete solution to the analytic solution as $h, k \rightarrow 0$ except in certain exceptional situations using spectral spatial discretisations.

The stability of discretisations of systems of o.d.e.'s with non-normal matrices has been a major research topic in the numerical analysis community in recent years $[8,9,11,12,13,14,15]$; A recent review article by van Dorsselaer et al [10] provides an excellent overview of these and many other references. The application is often to families of non-normal matrices arising from spatial discretisations of p.d.e.'s. Ideally, one would hope to prove strong stability,

$$
\left\|U^{(n)}\right\| \leq \gamma\left\|U^{(0)}\right\|
$$

with $\gamma$ being a constant which is not only independent of $n$ but is also a uniform bound applying to all matrices in the family of spatial discretisations for different mesh spacings $h$ but with the timestep $k$ being a function of $h$. One reason why strong stability is very desirable is that the Lax Equivalence Theorem proves that it is a necessary and sufficient condition for convergence of discrete solutions to the analytic solution on a finite time interval for all possible initial data, provided that the discretisation of the p.d.e. is consistent for sufficiently smooth initial data $[7]$.

At present, the conditions under which strong stability can be proved are too restrictive to be useful in practical computations. Instead, attention has focussed on weaker definitions of stability which are more easily achieved and are still useful for practical computations. One is algebraic stability $[8,11,12]$ which allows a linear growth in the transient solution of the form

$$
\left\|U^{(n)}\right\| \leq \gamma n\left\|U^{(0)}\right\|,
$$


where $\gamma$ is again a uniform constant. Another, due to Kreiss and Wu [9], is generalised stability which is based on exponentially weighted integrals over time for a inhomogeneous difference equation with homogeneous initial conditions. For both of these definitions, a sufficient condition for stability is that

$$
\tau(k C) \subset S,
$$

where the numerical range $\tau(k C)$ is a subset of the complex domain defined by

$$
\tau(k C)=\left\{k \frac{W^{*} C W}{W^{*} W}: W \neq 0\right\}
$$

where $W$ can be any non-zero complex vector of the required dimension and $W^{*}$ is its Hermitian, the complex conjugate transpose. The proof of sufficiency for algebraic stability is given by Lenferink and Spijker [12]. It proceeds in two parts, first showing that a certain resolvent condition is sufficient for algebraic stability, and then showing that this resolvent condition is satisfied if the numerical range lies inside $S$. Reddy and Trefethen [8] prove that the resolvent condition is necessary as well as sufficient for algebraic stability, and the equivalence to generalised stability follows almost immediately given the resolvent condition required by Kreiss and $\mathrm{Wu}[9]$.

By considering $W$ to be an eigenvector of $C$, it can be seen that $k \lambda \in \tau(k C)$ for each eigenvalue of $C$ and so the requirement that $\tau(k C) \subset S$ is a tighter restriction on the maximum allowable timestep than asymptotic stability. In comparison to strong stability, algebraic and generalised stability allow greater growth in transients when considering the solution behaviour as $t \rightarrow \infty$. On the finite time interval, it can be shown that under some very mild technical conditions they are sufficient for convergence of discrete solutions to the analytic solution as $h, k \rightarrow 0$ provided the initial data is smooth and the discretisation is consistent. It thus appears that these stability definitions are useful tools in analysing numerical discretisations, but additional research is still required.

In the Navier-Stokes application in this paper we will need to consider a slight generalisation to a system of o.d.e.'s of the form

$$
M \frac{d U}{d t}=C U
$$

in which $M$ is a real symmetric positive-definite matrix. The 'energy' is defined as $U^{*} M U$ which suggests the definition of new variables,

$$
V=M^{1 / 2} U
$$

so that $\|V\|^{2}=U^{*} M U$. If $M$ is diagonal then $M^{1 / 2}$ is the diagonal matrix whose elements are the positive square root of the corresponding elements of $M$. If $M$ is not diagonal then $M^{1 / 2}$ is equal to $T^{-1} \Lambda^{1 / 2} T$ where $\Lambda$ is the diagonal matrix of 
eigenvalues of $M$ and $T$ is the corresponding matrix of orthonormal eigenvectors. $T^{-1}=T^{*}$ and hence both $M^{1 / 2}$ and $M^{-1 / 2}$ are symmetric and positive definite.

Under the change of variables, the system of o.d.e.'s becomes

$$
\frac{d V}{d t}=M^{-1 / 2} C M^{-1 / 2} V
$$

which is algebraically stable provided $\tau\left(k M^{-1 / 2} C M^{-1 / 2}\right) \subset S$. If $C$ is either symmetric or anti-symmetric then so too is $M^{-1 / 2} C M^{-1 / 2}$ because of the symmetry of $M^{-1 / 2}$. Therefore, as discussed earlier the condition that the numerical range lies inside $S$ also ensures that the energy, $\|V\|^{2}=U^{*} M U$, will be non-increasing.

\section{Analytic equations}

The starting point for the analysis is the nonlinear Navier-Stokes equations,

$$
\frac{\partial U}{\partial t}+\frac{\partial F_{x}}{\partial x}+\frac{\partial F_{y}}{\partial y}+\frac{\partial F_{z}}{\partial z}=0
$$

$U$ is the vector of conservation variables $(\rho, \rho u, \rho v, \rho w, \rho E)^{T}$ and the flux terms are all defined in Appendix B together with the equation of state for an ideal gas and the definitions of the stress tensor and the viscous heat flux vector. The equations are to be solved on a unit cubic domain $\Omega$ with periodic boundary conditions. The choice of periodic b.c.'s avoids the complication of analysing the influence of different analytic and discrete boundary conditions.

The first step is to linearise the Navier-Stokes equations by considering perturbations to a steady flow which is uniform apart from spatial variations in the viscosity parameters $\mu, \lambda, k$. Perturbations to the conserved variables are related to the vector of primitive perturbations, $V=(\tilde{\rho}, \tilde{u}, \tilde{v}, \tilde{w}, \tilde{p})^{T}$, by the equation

$$
\widetilde{U}=R V \text {. }
$$

The uniform transformation matrix $R$ is given in Appendix B. Together, the linearisation and the change of variables yields

$$
\begin{aligned}
\frac{\partial V}{\partial t}+A_{x}^{\prime} \frac{\partial V}{\partial x}+A_{y}^{\prime} \frac{\partial V}{\partial y}+A_{z}^{\prime} \frac{\partial V}{\partial y} & =\frac{\partial}{\partial x}\left(D_{x x}^{\prime} \frac{\partial V}{\partial x}+D_{x y}^{\prime} \frac{\partial V}{\partial y}+D_{x z}^{\prime} \frac{\partial V}{\partial z}\right) \\
& +\frac{\partial}{\partial y}\left(D_{y x}^{\prime} \frac{\partial V}{\partial x}+D_{y y}^{\prime} \frac{\partial V}{\partial y}+D_{y z}^{\prime} \frac{\partial V}{\partial z}\right) \\
& +\frac{\partial}{\partial z}\left(D_{z x}^{\prime} \frac{\partial V}{\partial x}+D_{z y}^{\prime} \frac{\partial V}{\partial y}+D_{z z}^{\prime} \frac{\partial V}{\partial z}\right) .
\end{aligned}
$$

All matrices in this equation are listed in Appendix B. The second step is to define a further transformation of variables,

$$
V=S W
$$


The transformation matrix $S$, also given in Appendix $\mathrm{B}$, is due to Abarbanel and Gottlieb [16]. It has the property that the corresponding transformed equations,

$$
\begin{aligned}
\frac{\partial W}{\partial t}+A_{x} \frac{\partial W}{\partial x}+A_{y} \frac{\partial W}{\partial y}+A_{z} \frac{\partial W}{\partial y} & =\frac{\partial}{\partial x}\left(D_{x x} \frac{\partial W}{\partial x}+D_{x y} \frac{\partial W}{\partial y}+D_{x z} \frac{\partial W}{\partial z}\right) \\
& +\frac{\partial}{\partial y}\left(D_{y x} \frac{\partial W}{\partial x}+D_{y y} \frac{\partial W}{\partial y}+D_{y z} \frac{\partial W}{\partial z}\right) \\
& +\frac{\partial}{\partial z}\left(D_{z x} \frac{\partial W}{\partial x}+D_{z y} \frac{\partial W}{\partial y}+D_{z z} \frac{\partial W}{\partial z}\right)
\end{aligned}
$$

are such that the matrices $A_{x}, A_{y}, A_{z}$ and the combined dissipation matrix

$$
\left(\begin{array}{lll}
D_{x x} & D_{x y} & D_{x z} \\
D_{y x} & D_{y y} & D_{y z} \\
D_{z x} & D_{z y} & D_{z z}
\end{array}\right)
$$

are all symmetric. The matrices are listed in detail in Appendix B and it is also proved that the combined dissipation matrix is positive semi-definite provided that $\mu \geq 0,2 \mu+3 \lambda \geq 0$ and $k \geq 0$. These three conditions are satisfied by the laminar viscosity coefficients; it will be assumed that they are also satisfied by the coefficients defined by the turbulence modelling.

The perturbation 'energy' is defined as

$$
E=\int_{\Omega} \frac{1}{2} W^{*} W d V
$$

where $W^{*}$ again denotes the Hermitian of $W$, and its rate of change is

$$
\frac{d E}{d t}=\int_{\Omega} \frac{1}{2}\left(W^{*} \frac{\partial W}{\partial t}+\frac{\partial W^{*}}{\partial t} W\right) d V=\int_{\Omega} \frac{1}{2}\left(W^{*} \frac{\partial W}{\partial t}+\left(W^{*} \frac{\partial W}{\partial t}\right)^{*}\right) d V .
$$

Using the fact that $A_{x}$ is real and symmetric, and then integrating by parts using the periodic boundary conditions,

$$
\begin{gathered}
\int_{\Omega}\left(W^{*} A_{x} \frac{\partial W}{\partial x}\right)^{*} d V=\int_{\Omega} \frac{\partial W^{*}}{\partial x} A_{x} W d V=-\int_{\Omega} W^{*} A_{x} \frac{\partial W}{\partial x} d V \\
\Longrightarrow \quad \int_{\Omega} W^{*} A_{x} \frac{\partial W}{\partial x}+\left(W^{*} A_{x} \frac{\partial W}{\partial x}\right)^{*} d V=0 .
\end{gathered}
$$

Similarly,

$$
\begin{aligned}
& \int_{\Omega} W^{*} A_{y} \frac{\partial W}{\partial y}+\left(W^{*} A_{y} \frac{\partial W}{\partial y}\right)^{*} d V=0 \\
& \int_{\Omega} W^{*} A_{z} \frac{\partial W}{\partial z}+\left(W^{*} A_{z} \frac{\partial W}{\partial z}\right)^{*} d V=0 .
\end{aligned}
$$


Integrating the diffusion terms by parts and noting that

$$
\left[\left(\begin{array}{c}
\frac{\partial W}{\partial x} \\
\frac{\partial W}{\partial y} \\
\frac{\partial W}{\partial z}
\end{array}\right)^{*}\left(\begin{array}{ccc}
D_{x x} & D_{x y} & D_{x z} \\
D_{y x} & D_{y y} & D_{y z} \\
D_{z x} & D_{z y} & D_{z z}
\end{array}\right)\left(\begin{array}{c}
\frac{\partial W}{\partial x} \\
\frac{\partial W}{\partial y} \\
\frac{\partial W}{\partial z}
\end{array}\right)\right]^{*}=\left(\begin{array}{c}
\frac{\partial W}{\partial x} \\
\frac{\partial W}{\partial y} \\
\frac{\partial W}{\partial z}
\end{array}\right)^{*}\left(\begin{array}{ccc}
D_{x x} & D_{x y} & D_{x z} \\
D_{y x} & D_{y y} & D_{y z} \\
D_{z x} & D_{z y} & D_{z z}
\end{array}\right)\left(\begin{array}{c}
\frac{\partial W}{\partial x} \\
\frac{\partial W}{\partial y} \\
\frac{\partial W}{\partial z}
\end{array}\right)
$$

since the combined dissipation matrix is real and symmetric, yields the final result,

$$
\frac{d E}{d t}=-\int_{\Omega}\left(\begin{array}{c}
\frac{\partial W}{\partial x} \\
\frac{\partial W}{\partial y} \\
\frac{\partial W}{\partial z}
\end{array}\right)^{*}\left(\begin{array}{ccc}
D_{x x} & D_{x y} & D_{x z} \\
D_{y x} & D_{y y} & D_{y z} \\
D_{z x} & D_{z y} & D_{z z}
\end{array}\right)\left(\begin{array}{c}
\frac{\partial W}{\partial x} \\
\frac{\partial W}{\partial y} \\
\frac{\partial W}{\partial z}
\end{array}\right) d V
$$

Since the combined dissipation matrix is positive semi-definite, the perturbation 'energy' is non-increasing thereby proving stability in the energy norm.

\section{Semi-discrete equations}

Using an unstructured grid of tetrahedral cells with $W$ defined by linear interpolation between nodal values, the standard Galerkin spatial discretisation of the transformed p.d.e. is

$$
M_{G} \frac{d W}{d t}+A W=-D W
$$

where

$$
\begin{aligned}
m_{G_{i j}}= & \int_{\Omega} N_{i} N_{j} I d V \\
a_{i j}= & \int_{\Omega} N_{i}\left(A_{x} \frac{\partial N_{j}}{\partial x}+A_{y} \frac{\partial N_{j}}{\partial y}+A_{z} \frac{\partial N_{j}}{\partial x}\right) d V \\
d_{i j}= & \int_{\Omega}\left(D_{x x} \frac{\partial N_{i}}{\partial x} \frac{\partial N_{j}}{\partial x}+D_{x y} \frac{\partial N_{i}}{\partial x} \frac{\partial N_{j}}{\partial y}+D_{x z} \frac{\partial N_{i}}{\partial x} \frac{\partial N_{j}}{\partial z}\right. \\
& \quad+D_{y x} \frac{\partial N_{i}}{\partial y} \frac{\partial N_{j}}{\partial x}+D_{y y} \frac{\partial N_{i}}{\partial y} \frac{\partial N_{j}}{\partial y}+D_{y z} \frac{\partial N_{i}}{\partial y} \frac{\partial N_{j}}{\partial z} \\
& \left.\quad+D_{z x} \frac{\partial N_{i}}{\partial z} \frac{\partial N_{j}}{\partial x}+D_{z y} \frac{\partial N_{i}}{\partial z} \frac{\partial N_{j}}{\partial y}+D_{z z} \frac{\partial N_{i}}{\partial z} \frac{\partial N_{j}}{\partial z}\right) d V .
\end{aligned}
$$

The vector $W$ of discrete nodal variables has 5 -component subvectors $w_{i}$ at each node $i$. For a particular pair of nodes $i, j, m_{G_{i j}}, a_{i j}$ and $d_{i j}$ denote the corresponding $5 \times 5$ submatrices of the matrices $M_{G}, A$ and $D$, respectively. $N_{i}$ is the piecewise linear function which is equal to unity at node $i$ and zero at all other nodes, and the viscosity parameters $\mu, \lambda$ and $k$ within the dissipation matrices are defined to be constant on each tetrahedron. 
A standard modification is to 'mass-lump' the matrix $M_{G}$, turning it into a diagonal matrix $M$ with

$$
m_{i i}=\sum_{j} m_{G_{i j}}=\int_{\Omega} N_{i} I d V=V_{i} I
$$

where $V_{i}$ is the volume associated with node $i$, defined as one quarter of the sum of the volumes of the surrounding tetrahedra.

Another standard modification when interested in accelerating convergence to a steady-state solution, is to precondition the 'mass-lumped' matrix so that

$$
m_{i i}=\frac{V_{i}}{\Delta t_{i}} I
$$

The objective of this preconditioning is to use local timesteps, $\Delta t_{i}$, which are larger in large computational cells than in small ones, so that fewer iterations of the fully-discrete equations will be needed to converge to the steady-state solution to within some specified tolerance.

The matrix $A$ is antisymmetric since, integrating by parts,

$$
\begin{aligned}
a_{i j} & =-\int A_{x} \frac{\partial N_{i}}{\partial x} N_{j}+A_{y} \frac{\partial N_{i}}{\partial y} N_{j}+A_{z} \frac{\partial N_{i}}{\partial z} N_{j} d V \\
& =-\int N_{j}\left(A_{x}^{T} \frac{\partial N_{i}}{\partial x}+A_{y}^{T} \frac{\partial N_{i}}{\partial y}+A_{z}^{T} \frac{\partial N_{i}}{\partial z}\right) d V \\
& =-\left(a_{j i}\right)^{T} .
\end{aligned}
$$

The matrix $D$ is clearly symmetric. Furthermore, for any vector $W$,

$$
W^{*} D W=\int_{\Omega}\left(\begin{array}{c}
\frac{\partial W}{\partial x} \\
\frac{\partial W}{\partial y} \\
\frac{\partial W}{\partial z}
\end{array}\right)^{*}\left(\begin{array}{ccc}
D_{x x} & D_{x y} & D_{x z} \\
D_{y x} & D_{y y} & D_{y z} \\
D_{z x} & D_{z y} & D_{z z}
\end{array}\right)\left(\begin{array}{c}
\frac{\partial W}{\partial x} \\
\frac{\partial W}{\partial y} \\
\frac{\partial W}{\partial z}
\end{array}\right) d V
$$

where

$$
\begin{aligned}
\frac{\partial W}{\partial x} & =\sum_{i} \frac{\partial N_{i}}{\partial x} w_{i} \\
\frac{\partial W}{\partial y} & =\sum_{i} \frac{\partial N_{i}}{\partial y} w_{i} \\
\frac{\partial W}{\partial z} & =\sum_{i} \frac{\partial N_{i}}{\partial z} w_{i} .
\end{aligned}
$$

Since the combined dissipation matrix is positive semi-definite, it follows therefore that $D$ is also positive semi-definite. 
Defining the 'energy' for arbitrary complex $W$ as either $E=\frac{1}{2} W^{*} M_{G} W$ or $E=\frac{1}{2} W^{*} M W$, depending whether or not mass-lumping is used,

$$
\begin{aligned}
\frac{d E}{d t} & =-\frac{1}{2}\left(W^{*}(A+D) W+W^{*}(A+D)^{*} W\right) \\
& =-\frac{1}{2}\left(W^{*}(A+D) W+W^{*}(-A+D) W\right) \\
& =-W^{*} D W \leq 0
\end{aligned}
$$

and so the energy is non-increasing. Since both $M_{G}$ and $M$ are symmetric and positive definite this in turn implies stability for the semi-discrete equations.

Note that other discretisations of the Navier-Stokes equations will result in equations of the form,

$$
M \frac{d U}{d t}=C U
$$

where $M$ is a symmetric positive definite 'mass' matrix and $C$ can be decomposed into its symmetric and anti-symmetric components,

$$
C=-(A+D), \quad A=-\frac{1}{2}\left(C-C^{T}\right), \quad D=-\frac{1}{2}\left(C+C^{T}\right) .
$$

In general $A$ will now contain some terms due to the viscous discretisation, and $D$ will contain some terms due to the numerical smoothing associated with the convective discretisation. $D$ must still be positive semi-definite to ensure stability.

\section{$5 \quad$ Fully discrete equations}

Using Runge-Kutta time integration the fully discrete equations using one of the two diagonal mass matrices are

$$
W^{(n+1)}=L\left(k M^{-1} C\right) W^{(n)}
$$

where $L(z)$ is the Runge-Kutta polynomial with stability region $S$ as defined in Section 2 and $C=-(A+D)$. As explained in Section 2, sufficient conditions for algebraic and generalised stability are that

$$
\tau\left(k M^{-1 / 2} C M^{-1 / 2}\right) \subset S
$$

where

$$
\tau\left(k M^{-1 / 2} C M^{-1 / 2}\right)=\left\{-k \frac{W^{*} M^{-1 / 2} C M^{-1 / 2} W}{W^{*} W}: W \neq 0\right\} .
$$

For unsteady calculations with the diagonal mass-lumped matrix, the aim is simply to find the largest $k$ such that the constraint, Eq. (5.2), is satisfied. For steady-state calculations using the pre-conditioned mass matrix, one uses 
a pseudo-timestep $k=1$ and then the objective is to define the local timesteps $\Delta t_{i}$ to be as large as possible, again subject to the sufficient stability constraint, Eq. (5.2).

The difficulty is that direct evaluation of $\tau\left(k M^{-1 / 2} C M^{-1 / 2}\right)$ is not possible. Instead, a bounding set is constructed to enclose the numerical range and sufficient conditions are determined for this bounding set to lie inside $S$. There are two choices of bounding set which are relatively easily constructed, a halfdisk and a rectangle. The construction of the bounding half-disk starts with the observation that

$$
\left|\frac{W^{*} M^{-1 / 2} C M^{-1 / 2} W}{W^{*} W}\right| \leq\left\|M^{-1 / 2} C M^{-1 / 2}\right\| .
$$

Let the variable $r$ be defined by

$$
r=\max _{i}\left\{m_{i}^{-1} \max \left\{\sum_{j}\left\|c_{i j}\right\|, \sum_{j}\left\|c_{j i}\right\|\right\}\right\}
$$

where

$$
m_{i}= \begin{cases}V_{i}, & \text { mass-lumped matrix } \\ \frac{V_{i}}{\Delta t_{i}}, & \text { preconditioned mass-lumped matrix }\end{cases}
$$

Considering an arbitrary vector $V$, with subvector $v_{i}$ at each node $i$,

$$
\begin{aligned}
&\left\|M^{-1 / 2} C M^{-1 / 2} V\right\|^{2}=\sum_{i} m_{i}^{-1}\left|\sum_{j} c_{i j}\left(m_{j}^{-1 / 2} v_{j}\right)\right|^{2} \\
& \leq \sum_{i, j, k} m_{i}^{-1}\left\|c_{i j}\right\| m_{j}^{-1 / 2}\left\|v_{j}\right\|\left\|c_{i k}\right\| m_{k}^{-1 / 2}\left\|v_{k}\right\| \\
& \leq \sum_{i, j, k} m_{i}^{-1} m_{j}^{-1}\left\|v_{j}\right\|^{2}\left\|c_{i j}\right\|\left\|c_{i k}\right\| \\
& \leq r \sum_{i, j} m_{j}^{-1}\left\|v_{j}\right\|^{2}\left\|c_{i j}\right\| \\
& \leq r^{2}\|V\|^{2}, \\
& \Longrightarrow \quad\left\|M^{-1 / 2} C M^{-1 / 2}\right\| \leq r .
\end{aligned}
$$

The third line in the above derivation uses the inequality

$$
m_{j}^{-1 / 2}\left\|v_{j}\right\| m_{k}^{-1 / 2}\left\|v_{k}\right\| \leq \frac{1}{2}\left(m_{j}^{-1}\left\|v_{j}\right\|^{2}+m_{k}^{-1}\left\|v_{k}\right\|^{2}\right)
$$

followed by an interchange of subscripts to replace $m_{k}^{-1}\left\|v_{k}\right\|^{2}$ by $m_{j}^{-1}\left\|v_{j}\right\|^{2}$ given that $\left\|c_{i j}\right\|\left\|c_{i k}\right\|$ is symmetric in $j$ and $k$.

Also, for an arbitrary vector $W$,

$$
W^{*} C W+\left(W^{*} C W\right)^{*}=W^{*}\left(C+C^{*}\right) W=-2 W^{*} D W \leq 0
$$


and so the real component of $W^{*} C W$ must be zero or negative. Combined with the previous bound, this means that $\tau\left(k M^{-1 / 2} C M^{-1 / 2}\right)$ must therefore lie in the half-disk

$$
\{z=x+i y: x \leq 0,|z| \leq k r\} .
$$

For unsteady calculations, the necessary and sufficient condition for the halfdisk to lie inside $S$, and thus a sufficient condition for algebraic and generalised stability is

$$
k r \leq r_{c},
$$

where $r_{c}$ is the radius of the half-disk inscribing $S$, as defined and illustrated in Appendix A.

For preconditioned steady-state calculations with local timesteps, $k=1$ and so the largest value for $r$ for which the half-disk lies inside $S$ is $r_{c}$. For each node $i, \Delta t_{i}$ is then maximised subject to the definition of $r$ by

$$
\Delta t_{i}=\frac{r_{c} V_{i}}{\max \left\{\sum_{j}\left\|c_{i j}\right\|, \sum_{j}\left\|c_{j i}\right\|\right\}} .
$$

These stability limits require knowledge of $\left\|c_{i j}\right\|$. Appendix C evaluates $\left\|a_{i j}\right\|$ exactly, using the fact that it is a symmetric matrix. Since $a_{j i}=-a_{i j}^{T}=-a_{i j}$, it follows that $\left\|a_{j i}\right\|=\left\|a_{i j}\right\|$. Appendix $\mathrm{C}$ also constructs a tight upper bound for $\left\|d_{i j}\right\|$ and $\left\|d_{j i}\right\|$. From these, an upper bound for $\left\|c_{i j}\right\|$ is obtained. Replacing $\left\|c_{i j}\right\|$ by this upper bound in the above stability limits gives a new slightly more restrictive sufficient stability condition which can be easily evaluated.

This completes the use of the half-disk as a bounding set for the numerical range. The rectangular bounding set is obtained by considering separately the numerical ranges of $D$ and $A$.

Since $D$ is symmetric positive semidefinite, $x=-\frac{W^{*} M^{-1 / 2} D M^{-1 / 2} W}{W^{*} W}$ is real and negative with $-x_{d} \leq x \leq 0$ and $x_{d}$ defined by

$$
x_{d}=\max _{i}\left\{m_{i}^{-1} \max \left\{\sum_{j}\left\|d_{i j}\right\|, \sum_{j}\left\|d_{j i}\right\|\right\}\right\} \text {. }
$$

Similarly, since $A$ is anti-symmetric, $y=i \frac{W^{*} M^{-1 / 2} A M^{-1 / 2} W}{W^{*} W}$ is real and $|y| \leq y_{a}$ with $y_{a}$ defined by

$$
y_{a}=\max _{i}\left\{m_{i}^{-1} \max \left\{\sum_{j}\left\|a_{i j}\right\|, \sum_{j}\left\|a_{j i}\right\|\right\}\right\}=\max _{i}\left\{m_{i}^{-1} \sum_{j}\left\|a_{i j}\right\|\right\} .
$$

Thus the numerical range $\tau\left(k M^{-1 / 2} C M^{-1 / 2}\right)$ must lie inside the rectangle

$$
R=\left\{x+i y:-k x_{d} \leq x \leq 0,|y| \leq k y_{a}\right\} .
$$


For unsteady calculations, a sufficient stability limit is obtained by requiring that $R \subset S$. If the boundary of $S$ can be represented by $z=r \exp (i \theta)$ with $r(\theta)$ being a single-valued function for $\frac{\pi}{2} \leq \theta \leq \frac{3 \pi}{2}$ then this can written as

$$
k \sqrt{x_{d}^{2}+y_{a}^{2}} \leq r(\theta), \quad \tan (\theta)=-\frac{y_{a}}{x_{d}} .
$$

For preconditioned steady-state calculations, we again let $k=1$ and can then choose any rectangle $R$ which inscribes $S$. Appendix A shows the particular example of a half-square for which $x_{d}=y_{a}$. The maximum local timestep $\Delta t_{i}$ subject to the definitions of both $x_{D}$ and $y_{A}$ is then

$$
\Delta t_{i}=\min \left\{\frac{x_{d} V_{i}}{\max \left\{\sum_{j}\left\|d_{i j}\right\|, \sum_{j}\left\|d_{j i}\right\|\right\}}, \frac{y_{a} V_{i}}{\sum_{j}\left\|a_{i j}\right\|}\right\} .
$$

The final form of the stability limit is again obtained by using the results of Appendix $\mathrm{C}$ to evaluate $\left\|a_{i j}\right\|$ and place an upper bound on $\left\|d_{i j}\right\|$ and $\left\|d_{j i}\right\|$.

It is difficult to predict a priori which bounding set will give the least restrictive sufficient stability conditions. It depends in part on the particular RungeKutta method which is used. Appendix A shows that for some methods the inscribing half-disk almost contains the inscribing half-square and other rectangles lying inside $S$; in this case the half-disk sufficient stability conditions will probably be less restrictive. With other methods, the half-square almost contains the inscribing half-disk and for these the half-square stability conditions will probably be less restrictive.

In either case, the timestep limits are sufficient conditions for algebraic and generalised stability, but will almost certainly not be necessary. This point is well illustrated by considering the stability limits in the hyperbolic and parabolic extremes. In the hyperbolic case in which $D=0$, corresponding to a discretisation of the inviscid Euler equations, the best stability condition obtained from the analysis in this paper comes from an extreme limit of the rectangular bounding set. Setting $x_{d}=0$ and $y_{a}=r_{a}$, where $r_{a}$ is defined in Appendix A to be the length of the positive imaginary axis lying inside the stability region $S$, gives the local timestep stability limit

$$
\Delta t_{i} \leq \frac{r_{a} V_{i}}{\sum_{j}\left\|a_{i j}\right\|} .
$$

As explained in Section 2, because $A$ is anti-symmetric, this will also ensure that the 'energy' $W^{*} M W$ will be non-increasing. This represents a generalisation to arbitrary Runge-Kutta methods of the earlier energy analysis by Giles for two specific Runge-Kutta methods [6]. In that earlier work, the sufficient stability 
limit derived by energy analysis was compared to the necessary and sufficient Fourier stability limit for a uniform mesh. At worst, when the Mach number is zero and the grid spacing is the same in each direction, the timestep limit from the energy analysis is $40 \%$ less than that from the Fourier analysis. At best, at high Mach numbers or on stretched grids, the two timestep limits are almost equal.

In the parabolic case in which $A=0$, which would correspond to a simple diffusion problem, or the incompressible Navier-Stokes equations at a very low Reynolds number, the corresponding stability limit comes from setting $y_{a}=0$ and $x_{d}=r_{d}$, where $r_{d}$ is defined in Appendix A to be the length of the negative real axis lying inside the stability region $S$. The sufficient timestep stability limit is then

$$
\Delta t_{i} \leq \frac{r_{d} V_{i}}{\max \left\{\sum_{j}\left\|d_{i j}\right\|, \sum_{j}\left\|d_{j i}\right\|\right\}} .
$$

An ad hoc timestep limit which could perhaps be used comes from combining these last two limits to give

$$
\frac{1}{\Delta t_{i}^{2}}=\frac{1}{\Delta t_{a i}^{2}}+\frac{1}{\Delta t_{d i}^{2}}
$$

where $\Delta t_{a i}$ and $\Delta t_{d i}$ are the hyperbolic and parabolic timestep limits given by Eq. (5.17) and Eq. (5.18). It is possible to rigorously justify this combined limit if the grid is uniform, the viscous coefficients are uniform, and the stability region $S$ contains the half-ellipse passing through the points $i r_{a},-r_{d},-i r_{a}$. However, in general this timestep formulation can not be justified and so should only be used with care. Its advantage over the rigorous stability limits using the half-disk and the rectangle is that it will give a larger timestep which is hopefully still stable.

\section{Conclusions}

This paper has analysed the stability of one class of discretisations of the NavierStokes equations on a tetrahedral grid. The sufficient stability limits for both global and local timesteps are based on recent advances in numerical analysis. Additional research is needed to validate the usefulness of these limits, whether they are close enough to the necessary stability limits to be a valuable practical criterion on which to base the timestep in actual computations.

Another direction for future research is the extension of the analysis to other discretisations. Upwind approximations of the inviscid fluxes would be a particularly interesting topic for study. As indicated at the end of Section 4, this would change the definition of the dissipation matrix $D$, but the overall approach to the stability analysis would remain valid. It may also be possible to investigate the stability of different Navier-Stokes boundary condition implementations by incorporating these within the coupled system of o.d.e.'s. 


\section{Acknowledgements}

I wish to thank Larry Wigton for stimulating this research and Eli Turkel, Eitan Tadmor, Bill Morton, Endré Süli, Nick Trefethen and Satish Reddy for their help with the numerical analysis literature on the stability of systems of o.d.e.'s with non-normal matrices, and for their valuable comments on the paper. The financial support of Rolls-Royce plc, DTI and EPSRC is gratefully acknowledged.

\section{References}

[1] L. Wigton. Personal communication, 1994.

[2] N.P. Weatherill, O. Hassan, M.J. Marchant, and D.L. Marcum. Adaptive inviscid flow solutions for aerospace geometries on efficiently generated unstructured tetrahedral meshes. AIAA Paper 93-3390, 1993.

[3] J. Peraire, J. Peiró, and K. Morgan. Finite element multigrid solution of Euler flows past installed aero-engines. Comput. Mech., 11:433-451, 1993.

[4] R.D. Rausch, J.T. Batina, and H.T.Y. Yang. Three-dimensional timemarching aeroelastic analyses using an unstructured-grid Euler method. AIAA J., 31(9):1626-1633, 1993.

[5] P. Crumpton and M.B. Giles. Aircraft computations using multigrid and an unstructured parallel library. AIAA Paper 95-0210, 1995.

[6] M.B. Giles. Energy stability analysis of multi-step methods on unstructured meshes. Technical Report TR-87-1, MIT Dept. of Aero. and Astro., 1987.

[7] R.D. Richtmyer and K.W. Morton. Difference Methods for Initial Value Problems. Wiley-Interscience, 2nd edition, 1967.

[8] S.C. Reddy and L.N. Trefethen. Stability of the method of lines. Numer. Math., 62:235-267, 1992.

[9] H.O. Kreiss and L. Wu. On the stability definition of difference approximations for the initial boundary value problem. Appl. Num. Math., 12:213-227, 1993.

[10] J.L.M. van Dorsselaer, J.F.B Kraaijevanger, and M.N. Spijker. Linear stability analysis in the numerical solution of initial value problems. Acta Numerica, pages 199-237, 1993.

[11] J.F.B.M. Kraaijevanger, H.W.J. Lenferink, and M.N. Spijker. Stepsize restrictions for stability in the numerical solution of ordinary and partial differential equations. J. Comput. Appl. Math., 20:67-81, Nov 1987. 
[12] H.W.J. Lenferink and M.N. Spijker. On the use of stability regions in the numerical analysis of initial value problems. Math. Comp., 57(195):221-237, 1991.

[13] S.C. Reddy and L.N. Trefethen. Lax-stability of fully discrete spectral methods via stability regions and pseudo-eigenvalues. Comput. Methods Appl. Mech. Engrg., 80:147-164, 1990.

[14] S.C. Reddy. Pseudospectra of Operators and Discretization Matrices and an Application to Stability of the Method of Lines. PhD thesis, Massachusetts Institute of Technology, Cambridge, Massachusetts 02139, 1991. Numerical Analysis Report 91-4.

[15] C. Lubich and O. Nevanlinna. On resolvent conditions and stability estimates. BIT, 31:293-313, 1991.

[16] S. Abarbanel and D. Gottlieb. Optimal time splitting for two- and threedimensional Navier-Stokes equations with mixed derivatives. Journal of Computational Physics, 35:1-33, 1981. 


\section{Appendix A Runge-Kutta stability curves}

An example of a Runge-Kutta type of approximation of the o.d.e.

$$
\frac{d u}{d t}=\lambda u
$$

is the following two-stage predictor-corrector method,

$$
\begin{aligned}
u^{(1)} & =u^{n}+k \lambda u^{n} \\
u^{n+1} & =u^{n}+k \lambda u^{(1)} .
\end{aligned}
$$

Combining these two equations gives

$$
u^{n+1}=L(k \lambda) u^{n},
$$

where the Runge-Kutta polynomial function is $L(z)=1+z+z^{2}$. Figure 1a) shows the stability region $S$ within which $|L| \leq 1$. It also shows the largest half-disk,

$$
\left\{z=x+i y: x \leq 0,|z| \leq r_{c}\right\},
$$

and the largest half-square,

$$
\left\{z=x+i y:-\frac{r_{s}}{\sqrt{2}} \leq x \leq 0,|y| \leq \frac{r_{s}}{\sqrt{2}}\right\},
$$

which lie inside $S$. If the boundary of $S$ is defined as $z=r \exp (i \theta)$ then $r_{c}$ and $r_{s}$ can be defined as

$$
r_{c}=\min _{\frac{\pi}{2} \leq \theta \leq \frac{3 \pi}{2}} r(\theta), \quad r_{s}=r\left(\frac{3}{4} \pi\right) .
$$

The values of $r_{c}$ and $r_{s}$ are listed to the right of the figure along with those of two other important parameters, $r_{a}=r\left(\frac{1}{2} \pi\right)$, which is the length of the positive imaginary axis segment within $S$, and $r_{d}=r(\pi)$, which is the length of the negative real axis segment within $S$. The importance of all four of these parameters is discussed in the main text in Section 5.

Figures 1b) and 1c) show the corresponding curves and data for two other popular multistage integration schemes. 


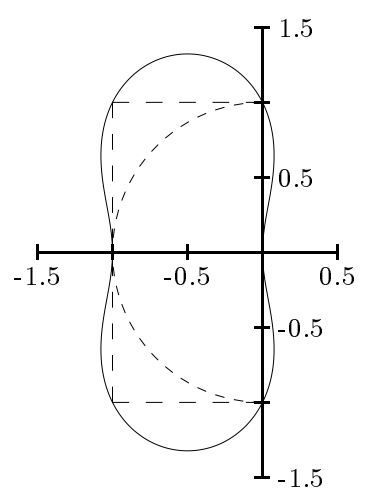

a) Predictor-corrector

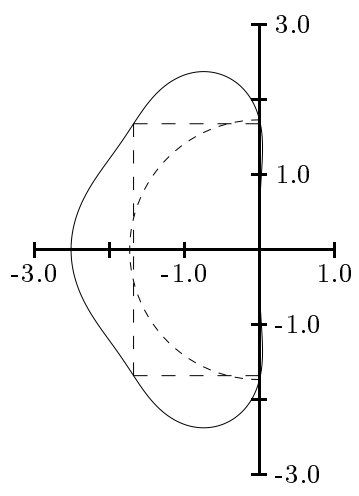

$$
\begin{aligned}
u^{(1)} & =u^{n}+\lambda \Delta t u^{n} \\
u^{n+1} & =u^{n}+\lambda \Delta t u^{(1)} \\
r_{c} & =1.0 \\
r_{s} & =1.414 \\
r_{a} & =1.0 \\
r_{d} & =1.0
\end{aligned}
$$

b) Three-stage scheme

$$
\begin{aligned}
u^{(1)} & =u^{n}+\frac{1}{3} \lambda \Delta t u^{n} \\
u^{(2)} & =u^{n}+\frac{1}{2} \lambda \Delta t u^{(1)} \\
u^{n+1} & =u^{n}+\lambda \Delta t u^{(2)} \\
r_{c} & =1.731 \\
r_{s} & =2.375 \\
r_{a} & =1.731 \\
r_{d} & =2.513
\end{aligned}
$$

c) Four-stage scheme

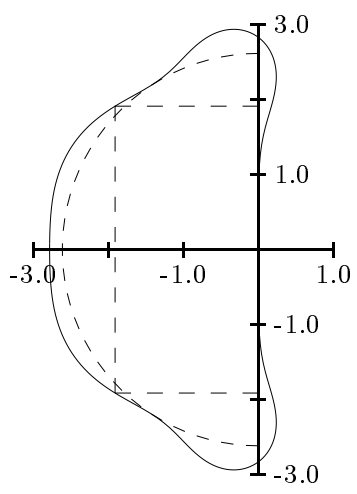

$$
\begin{aligned}
u^{(1)} & =u^{n}+\frac{1}{4} \lambda \Delta t u^{n} \\
u^{(2)} & =u^{n}+\frac{1}{3} \lambda \Delta t u^{(1)} \\
u^{(3)} & =u^{n}+\frac{1}{2} \lambda \Delta t u^{(2)} \\
u^{n+1} & =u^{n}+\lambda \Delta t u^{(3)} \\
r_{c} & =2.616 \\
r_{s} & =2.704 \\
r_{a} & =2.828 \\
r_{d} & =2.785
\end{aligned}
$$

Figure 1: Stability boundary and inscribing half-disk and half-square for three Runge-Kutta methods 


\section{Appendix B vectors, matrices and positivity}

Starting with the conservative form of the Navier-Stokes equations, the state vector and flux vectors are

$$
\begin{aligned}
& U=\left(\begin{array}{l}
\rho \\
\rho u \\
\rho v \\
\rho w \\
\rho E
\end{array}\right) \\
& F_{x}=\left(\begin{array}{ll}
\rho u & \\
\rho u^{2}+p & -\tau_{x x} \\
\rho u v & -\tau_{y x} \\
\rho u w & -\tau_{z x} \\
\rho u\left(E+\frac{p}{\rho}\right) & -u \tau_{x x}-v \tau_{y w}-w \tau_{z x}+q_{x}
\end{array}\right) \\
& F_{y}=\left(\begin{array}{ll}
\rho v & -\tau_{x y} \\
\rho u v & -\tau_{y y} \\
\rho v^{2}+p & -\tau_{z y} \\
\rho v w & -u \tau_{x y}-v \tau_{y y}-w \tau_{z y}+q_{y}
\end{array}\right) \\
& F_{z}=\left(\begin{array}{ll}
\rho w & \\
\rho u w & -\tau_{x z} \\
\rho v w & -\tau_{y z} \\
\rho w^{2}+p & -\tau_{z z} \\
\rho w\left(E+\frac{p}{\rho}\right) & -u \tau_{x z}-v \tau_{y z}-w \tau_{z z}+q_{z}
\end{array}\right) .
\end{aligned}
$$

$\rho, u, v, w, p, E$ are the density, three Cartesian velocity components, pressure and total internal energy, respectively. To complete the system of equations requires an equation of state for an ideal gas,

$$
p=\rho R T=(\gamma-1) \rho\left(E-\frac{1}{2}\left(u^{2}+v^{2}+w^{2}\right)\right),
$$

in which $R, T, \gamma$ are the gas constant, temperature and uniform specific heat ratio, respectively, as well as equations defining the heat fluxes,

$$
q_{x}=-k \frac{\partial T}{\partial x}, \quad q_{y}=-k \frac{\partial T}{\partial y}, \quad q_{z}=-k \frac{\partial T}{\partial z}
$$


and the viscous stress terms,

$$
\begin{array}{ll}
\tau_{x x}=2 \mu \frac{\partial u}{\partial x}+\lambda\left(\frac{\partial u}{\partial x}+\frac{\partial v}{\partial y}+\frac{\partial w}{\partial z}\right), & \tau_{x y}=\tau_{y x}=\mu\left(\frac{\partial u}{\partial y}+\frac{\partial v}{\partial x}\right) \\
\tau_{y y}=2 \mu \frac{\partial v}{\partial y}+\lambda\left(\frac{\partial u}{\partial x}+\frac{\partial v}{\partial y}+\frac{\partial w}{\partial z}\right), & \tau_{x z}=\tau_{z x}=\mu\left(\frac{\partial u}{\partial z}+\frac{\partial w}{\partial x}\right) \\
\tau_{z z}=2 \mu \frac{\partial w}{\partial z}+\lambda\left(\frac{\partial u}{\partial x}+\frac{\partial v}{\partial y}+\frac{\partial w}{\partial z}\right), & \tau_{y z}=\tau_{z y}=\mu\left(\frac{\partial v}{\partial z}+\frac{\partial w}{\partial y}\right) .
\end{array}
$$

The transformation from conservative to primitive variables, $(\rho u v w p)^{T}$, is accomplished by the matrix

$$
R=\left(\begin{array}{ccccc}
1 & 0 & 0 & 0 & 0 \\
u & \rho & 0 & 0 & 0 \\
v & 0 & \rho & 0 & 0 \\
w & 0 & 0 & \rho & 0 \\
\frac{u^{2}+v^{2}+w^{2}}{2} & \rho u & \rho v & \rho w & \frac{1}{\gamma-1}
\end{array}\right)
$$

The linearised, transformed equations are

$$
\begin{aligned}
\frac{\partial V}{\partial t}+A_{x}^{\prime} \frac{\partial V}{\partial x}+A_{y}^{\prime} \frac{\partial V}{\partial y}+A_{z}^{\prime} \frac{\partial V}{\partial z} & =\frac{\partial}{\partial x}\left(D_{x x}^{\prime} \frac{\partial V}{\partial x}+D_{x y}^{\prime} \frac{\partial V}{\partial y}+D_{x z}^{\prime} \frac{\partial V}{\partial z}\right) \\
& +\frac{\partial}{\partial y}\left(D_{y x}^{\prime} \frac{\partial V}{\partial x}+D_{y y}^{\prime} \frac{\partial V}{\partial y}+D_{y z}^{\prime} \frac{\partial V}{\partial z}\right) \\
& +\frac{\partial}{\partial z}\left(D_{z x}^{\prime} \frac{\partial U}{\partial x}+D_{z y}^{\prime} \frac{\partial V}{\partial y}+D_{z z}^{\prime} \frac{\partial V}{\partial z}\right)
\end{aligned}
$$

where

$$
\begin{aligned}
A_{x}^{\prime} & =\left(\begin{array}{ccccc}
u & \rho & 0 & 0 & 0 \\
0 & u & 0 & 0 & \frac{1}{\rho} \\
0 & 0 & u & 0 & 0 \\
0 & 0 & 0 & u & 0 \\
0 & \gamma p & 0 & 0 & u
\end{array}\right), \quad A_{y}^{\prime}=\left(\begin{array}{ccccc}
v & 0 & \rho & 0 & 0 \\
0 & v & 0 & 0 & 0 \\
0 & 0 & v & 0 & \frac{1}{\rho} \\
0 & 0 & 0 & v & 0 \\
0 & 0 & \gamma p & 0 & v
\end{array}\right) \\
A_{z}^{\prime} & =\left(\begin{array}{ccccc}
w & 0 & 0 & \rho & 0 \\
0 & w & 0 & 0 & 0 \\
0 & 0 & w & 0 & 0 \\
0 & 0 & 0 & w & \frac{1}{\rho} \\
0 & 0 & 0 & \gamma p & w
\end{array}\right)
\end{aligned}
$$


and

$$
\begin{aligned}
& D_{x x}^{\prime}=\left(\begin{array}{ccccc}
0 & 0 & 0 & 0 & 0 \\
0 & \frac{2 \mu+\lambda}{\rho} & 0 & 0 & 0 \\
0 & 0 & \frac{\mu}{\rho} & 0 & 0 \\
0 & 0 & 0 & \frac{\mu}{\rho} & 0 \\
-\frac{\gamma \mu p}{\operatorname{Pr} \rho^{2}} & 0 & 0 & 0 & \frac{\gamma \mu}{\operatorname{Pr} \rho}
\end{array}\right), \quad D_{x y}^{\prime}=D_{y x}^{\prime T}=\left(\begin{array}{ccccc}
0 & 0 & 0 & 0 & 0 \\
0 & 0 & \frac{\lambda}{\rho} & 0 & 0 \\
0 & \frac{\mu}{\rho} & 0 & 0 & 0 \\
0 & 0 & 0 & 0 & 0 \\
0 & 0 & 0 & 0 & 0
\end{array}\right) \\
& D_{y y}^{\prime}=\left(\begin{array}{ccccc}
0 & 0 & 0 & 0 & 0 \\
0 & \frac{\mu}{\rho} & 0 & 0 & 0 \\
0 & 0 & \frac{2 \mu+\lambda}{\rho} & 0 & 0 \\
0 & 0 & 0 & \frac{\mu}{\rho} & 0 \\
-\frac{\gamma \mu p}{\operatorname{Pr} \rho^{2}} & 0 & 0 & 0 & \frac{\gamma \mu}{\operatorname{Pr} \rho}
\end{array}\right), \quad D_{x z}^{\prime}=D_{z x}^{\prime T}=\left(\begin{array}{ccccc}
0 & 0 & 0 & 0 & 0 \\
0 & 0 & 0 & \frac{\lambda}{\rho} & 0 \\
0 & 0 & 0 & 0 & 0 \\
0 & \frac{\mu}{\rho} & 0 & 0 & 0 \\
0 & 0 & 0 & 0 & 0
\end{array}\right) \\
& D_{z z}^{\prime}=\left(\begin{array}{ccccc}
0 & 0 & 0 & 0 & 0 \\
0 & \frac{\mu}{\rho} & 0 & 0 & 0 \\
0 & 0 & \underline{\mu} & 0 & 0 \\
0 & 0 & 0 & \frac{2 \mu+\lambda}{\rho} & 0 \\
-\frac{\gamma \mu p}{\operatorname{Pr} \rho^{2}} & 0 & 0 & 0 & \frac{\gamma \mu}{\operatorname{Pr} \rho}
\end{array}\right), \quad D_{y z}^{\prime}=D_{z y}^{\prime T}=\left(\begin{array}{ccccc}
0 & 0 & 0 & 0 & 0 \\
0 & 0 & 0 & 0 & 0 \\
0 & 0 & 0 & \frac{\lambda}{\rho} & 0 \\
0 & 0 & \frac{\mu}{\rho} & 0 & 0 \\
0 & 0 & 0 & 0 & 0
\end{array}\right)
\end{aligned}
$$

The Prandtl number is defined as

$$
\operatorname{Pr}=\frac{\mu c_{p}}{k}=\frac{\gamma \mu R}{(\gamma-1) k},
$$

but is not assumed to be uniform since $\lambda$ and $k$ in general represent combinations of laminar and turbulent viscosities, each with their own Prandtl number.

The second transformation matrix is

$$
S=\left(\begin{array}{ccccc}
\sqrt{\gamma} \frac{\rho}{c} & 0 & 0 & 0 & 0 \\
0 & 1 & 0 & 0 & 0 \\
0 & 0 & 1 & 0 & 0 \\
0 & 0 & 0 & 1 & 0 \\
\frac{1}{\sqrt{\gamma}} \rho c & 0 & 0 & 0 & \sqrt{\frac{\gamma-1}{\gamma}} \rho c
\end{array}\right)
$$

and the transformed matrices are 


$$
\begin{aligned}
& A_{x}=S^{-1} A_{x}^{\prime} S=\left(\begin{array}{ccccc}
u & \frac{1}{\sqrt{\gamma}} c & 0 & 0 & 0 \\
\frac{1}{\sqrt{\gamma}} c & u & 0 & 0 & \sqrt{\frac{\gamma-1}{\gamma}} c \\
0 & 0 & u & 0 & 0 \\
0 & 0 & 0 & u & 0 \\
0 & \sqrt{\frac{\gamma-1}{\gamma}} c & 0 & 0 & u
\end{array}\right), \\
& A_{y}=S^{-1} A_{y}^{\prime} S=\left(\begin{array}{ccccc}
v & 0 & \frac{1}{\sqrt{\gamma}} c & 0 & 0 \\
0 & v & 0 & 0 & 0 \\
\frac{1}{\sqrt{\gamma}} c & 0 & v & 0 & \sqrt{\frac{\gamma-1}{\gamma}} c \\
0 & 0 & 0 & v & 0 \\
0 & 0 & \sqrt{\frac{\gamma-1}{\gamma}} c & 0 & v
\end{array}\right), \\
& A_{z}=S^{-1} A_{z}^{\prime} S=\left(\begin{array}{ccccc}
w & 0 & 0 & \frac{1}{\sqrt{\gamma}} c & 0 \\
0 & w & 0 & 0 & 0 \\
0 & 0 & w & 0 & 0 \\
\frac{1}{\sqrt{\gamma}} c & 0 & 0 & w & \sqrt{\frac{\gamma-1}{\gamma}} c \\
0 & 0 & 0 & \sqrt{\frac{\gamma-1}{\gamma}} c & w
\end{array}\right),
\end{aligned}
$$

and

$$
\begin{aligned}
& D_{x x}=\left(\begin{array}{ccccc}
0 & 0 & 0 & 0 & 0 \\
0 & \frac{2 \mu+\lambda}{\rho} & 0 & 0 & 0 \\
0 & 0 & \underline{\mu} & 0 & 0 \\
0 & 0 & 0 & \frac{\mu}{\rho} & 0 \\
0 & 0 & 0 & 0 & \frac{\gamma \mu}{\operatorname{Pr} \rho}
\end{array}\right), \quad D_{x y}=D_{y x}^{T}=\left(\begin{array}{ccccc}
0 & 0 & 0 & 0 & 0 \\
0 & 0 & \frac{\lambda}{\rho} & 0 & 0 \\
0 & \underline{\mu} & 0 & 0 & 0 \\
0 & 0 & 0 & 0 & 0 \\
0 & 0 & 0 & 0 & 0
\end{array}\right), \\
& D_{y y}=\left(\begin{array}{ccccc}
0 & 0 & 0 & 0 & 0 \\
0 & \frac{\mu}{\rho} & 0 & 0 & 0 \\
0 & 0 & \frac{2 \mu+\lambda}{\rho} & 0 & 0 \\
0 & 0 & 0 & \frac{\mu}{\rho} & 0 \\
0 & 0 & 0 & 0 & \frac{\gamma \mu}{\operatorname{Pr} \rho}
\end{array}\right), \quad D_{x z}=D_{z x}^{T}=\left(\begin{array}{ccccc}
0 & 0 & 0 & 0 & 0 \\
0 & 0 & 0 & \frac{\lambda}{\rho} & 0 \\
0 & 0 & 0 & 0 & 0 \\
0 & \frac{\mu}{\rho} & 0 & 0 & 0 \\
0 & 0 & 0 & 0 & 0
\end{array}\right),
\end{aligned}
$$




$$
D_{z z}=\left(\begin{array}{ccccc}
0 & 0 & 0 & 0 & 0 \\
0 & \underline{\mu} & 0 & 0 & 0 \\
0 & 0 & \frac{\mu}{\rho} & 0 & 0 \\
0 & 0 & 0 & \frac{2 \mu+\lambda}{\rho} & 0 \\
0 & 0 & 0 & 0 & \frac{\gamma \mu}{\operatorname{Pr} \rho}
\end{array}\right), \quad D_{y z}=D_{z y}^{T}=\left(\begin{array}{ccccc}
0 & 0 & 0 & 0 & 0 \\
0 & 0 & 0 & 0 & 0 \\
0 & 0 & 0 & \frac{\lambda}{\rho} & 0 \\
0 & 0 & \frac{\mu}{\rho} & 0 & 0 \\
0 & 0 & 0 & 0 & 0
\end{array}\right)
$$

An important feature of the transformed equations is that the combined dissipation matrix,

$$
\left(\begin{array}{ccc}
D_{x x} & D_{x y} & D_{x z} \\
D_{y x} & D_{y y} & D_{y z} \\
D_{z x} & D_{z y} & D_{z z}
\end{array}\right)
$$

is both symmetric and positive semi-definite. The symmetry is clear from the above definitions of the component matrices, and the positivity comes from noting that

$$
\begin{aligned}
x^{T}\left(\begin{array}{ccc}
D_{x x} & D_{x y} & D_{x z} \\
D_{y x} & D_{y y} & D_{y z} \\
D_{z x} & D_{z y} & D_{z z}
\end{array}\right) x & =\frac{\mu}{\rho}\left(x_{3}+x_{7}\right)^{2}+\frac{\mu}{\rho}\left(x_{4}+x_{12}\right)^{2}+\frac{\mu}{\rho}\left(x_{9}+x_{13}\right)^{2} \\
& +\frac{1}{\rho}\left(\begin{array}{c}
x_{2} \\
x_{8} \\
x_{14}
\end{array}\right)^{T}\left(\begin{array}{ccc}
2 \mu+\lambda & \lambda & \lambda \\
\lambda & 2 \mu+\lambda & \lambda \\
\lambda & \lambda & 2 \mu+\lambda
\end{array}\right)\left(\begin{array}{c}
x_{2} \\
x_{8} \\
x_{14}
\end{array}\right) \\
& +\frac{\gamma \mu}{\operatorname{Pr} \rho}\left(x_{5}^{2}+x_{10}^{2}+x_{15}^{2}\right) .
\end{aligned}
$$

The eigenvalues of

$$
\left(\begin{array}{ccc}
2 \mu+\lambda & \lambda & \lambda \\
\lambda & 2 \mu+\lambda & \lambda \\
\lambda & \lambda & 2 \mu+\lambda
\end{array}\right)
$$

are $2 \mu, 2 \mu, 2 \mu+3 \lambda$ and hence the combined dissipation matrix is positive semidefinite provided $\mu \geq 0,2 \mu+3 \lambda \geq 0$ and $k \geq 0$. 


\section{Appendix C $\quad L_{2}$ norms of component matrices}

Defining

$$
\int_{\Omega} N_{i} \nabla N_{j} d V=S \vec{n}
$$

then

$$
\begin{aligned}
a_{i j}=S\left(n_{x} A_{x}+n_{y} A_{y}+n_{z} A_{z}\right) \\
=S\left(\begin{array}{ccccc}
\vec{u} \cdot \vec{n} & \frac{1}{\sqrt{\gamma}} c n_{x} & \frac{1}{\sqrt{\gamma}} c n_{y} & \frac{1}{\sqrt{\gamma}} c n_{z} & 0 \\
\frac{1}{\sqrt{\gamma}} c n_{x} & \vec{u} \cdot \vec{n} & 0 & 0 & \sqrt{\frac{\gamma-1}{\gamma}} c n_{x} \\
\frac{1}{\sqrt{\gamma}} c n_{y} & 0 & \vec{u} \cdot \vec{n} & 0 & \sqrt{\frac{\gamma-1}{\gamma}} c n_{y} \\
\frac{1}{\sqrt{\gamma}} c n_{z} & 0 & 0 & \vec{u} \cdot \vec{n} & \sqrt{\frac{\gamma-1}{\gamma}} c n_{z} \\
0 & \sqrt{\frac{\gamma-1}{\gamma}} c n_{x} & \sqrt{\frac{\gamma-1}{\gamma}} c n_{y} & \sqrt{\frac{\gamma-1}{\gamma}} c n_{z} & \vec{u} \cdot \vec{n}
\end{array}\right) .
\end{aligned}
$$

Three of the eigenvalues of $S^{-1} a_{i j}$ are equal to $\vec{u} \cdot \vec{n}$ and the other two are $\vec{u} \cdot \vec{n} \pm c$. Hence,

$$
\left\|a_{i j}\right\|=S(|\vec{u} \cdot \vec{n}|+c)
$$

using the fact that for symmetric matrices the $L_{2}$ norm is the magnitude of the largest eigenvalue.

The quantity $S \vec{n}$ can be interpreted geometrically. First note that $\nabla N_{j}$ is non-zero only on tetrahedra surrounding node $j$, and that on such a tetrahedron, labelled $\sigma$,

$$
\nabla N_{j}=\frac{1}{3 V^{\sigma}} \vec{S}_{j}^{\sigma}
$$

where $\vec{S}_{j}^{\sigma}$ is the inward-pointing area vector of the face of $\sigma$ opposite node $j$, and $V^{\sigma}$ is the volume of the tetrahedron. Summing over all tetrahedra for which both $i$ and $j$ are corner nodes, gives

$$
S \vec{n}=\frac{1}{12} \sum_{\sigma} \vec{S}_{j}^{\sigma}
$$

Define $d_{i j}^{\sigma}$ to be the contribution to $d_{i j}$ from the integration over tetrahedron $\sigma$. Therefore,

$$
d_{i j}=\sum_{\sigma} d_{i j}^{\sigma} \quad \Longrightarrow \quad\left\|d_{i j}\right\|_{2} \leq \sum_{\sigma}\left\|d_{i j}^{\sigma}\right\|_{2}
$$

where again the summation is over tetrahedra common to both $i$ and $j$. On 
tetrahedron $\sigma, \nabla N_{i}$ and $\nabla N_{j}$ are both uniform and so

$$
d_{i j}^{\sigma}=V^{\sigma}\left(\begin{array}{ccccc}
0 & 0 & 0 & 0 & 0 \\
0 & \frac{\mu+\lambda}{\rho} \frac{\partial N_{i}}{\partial x} \frac{\partial N_{j}}{\partial x} & \frac{\mu+\lambda}{\rho} \frac{\partial N_{i}}{\partial x} \frac{\partial N_{j}}{\partial y} & \frac{\mu+\lambda}{\rho} \frac{\partial N_{i}}{\partial x} \frac{\partial N_{j}}{\partial z} & 0 \\
& +\frac{\mu}{\rho} \nabla N_{i} \cdot \nabla N_{j} & & \\
0 & \frac{\mu+\lambda}{\rho} \frac{\partial N_{i}}{\partial y} \frac{\partial N_{j}}{\partial x} & \frac{\mu+\lambda}{\rho} \frac{\partial N_{i}}{\partial y} \frac{\partial N_{j}}{\partial y} & \frac{\mu+\lambda}{\rho} \frac{\partial N_{i}}{\partial y} \frac{\partial N_{j}}{\partial z} & 0 \\
0 & \frac{\mu+\lambda}{\rho} \frac{\mu N_{i}}{\partial z} \frac{\partial N_{j}}{\partial x} & \frac{\mu+\lambda}{\rho} \frac{\partial N_{i}}{\partial z} \frac{\partial N_{j}}{\partial y} & \frac{\mu+\lambda}{\rho} \frac{\partial N_{i}}{\partial z} \frac{\partial N_{j}}{\partial z} & 0 \\
0 & 0 & 0 & \frac{\mu}{\rho} \nabla N_{i} \cdot \nabla N_{j} & \\
0 & 0 & 0 & \frac{\gamma \mu}{\operatorname{Pr} \rho} \nabla N_{i} \cdot \nabla N_{j}
\end{array}\right)
$$

Hence,

$$
\left\|d_{i j}^{\sigma}\right\| \leq V^{\sigma} \max \left\{\frac{\mu}{\rho}\left|\nabla N_{i} \cdot \nabla N_{j}\right|+\frac{\mu+\lambda}{\rho}\left|\nabla N_{i}\right|\left|\nabla N_{j}\right|, \frac{\gamma \mu}{\operatorname{Pr} \rho}\left|\nabla N_{i} \cdot \nabla N_{j}\right|\right\}
$$

which can be re-expressed using the values for $\nabla N_{i}$ and $\nabla N_{j}$ as

$$
\left\|d_{i j}^{\sigma}\right\| \leq \frac{1}{9 V^{\sigma}} \max \left\{\frac{\mu}{\rho}\left|\vec{S}_{i}^{\sigma} \cdot \vec{S}_{j}^{\sigma}\right|+\frac{\mu+\lambda}{\rho}\left|\vec{S}_{i}^{\sigma}\right|\left|\vec{S}_{j}^{\sigma}\right|, \frac{\gamma \mu}{\operatorname{Pr} \rho}\left|\vec{S}_{i}^{\sigma} \cdot \vec{S}_{j}^{\sigma}\right|\right\},
$$

where $\vec{S}_{i}^{\sigma}$ and $\vec{S}_{j}^{\sigma}$ are as defined previously. Note that the upper bound on the right-hand-side of Eq. (C.9) is unchanged if $i$ and $j$ are interchanged, and so it is also an upper bound for $\left\|d_{j i}^{\sigma}\right\|$. Hence,

$$
\max \left\{\left\|d_{i j}\right\|,\left\|d_{j i}\right\|\right\} \leq \sum_{\sigma} \frac{1}{9 V^{\sigma}} \max \left\{\frac{\mu}{\rho}\left|\vec{S}_{i}^{\sigma} \cdot \vec{S}_{j}^{\sigma}\right|+\frac{\mu+\lambda}{\rho}\left|\vec{S}_{i}^{\sigma}\right|\left|\vec{S}_{j}^{\sigma}\right|, \frac{\gamma \mu}{\operatorname{Pr} \rho}\left|\vec{S}_{i}^{\sigma} \cdot \vec{S}_{j}^{\sigma}\right|\right\}
$$

The exact value for $\left\|a_{i j}\right\|$ and the upper bounds for $\left\|d_{i j}\right\|,\left\|d_{j i}\right\|$ can then be combined by the triangle inequality,

$$
\left\|c_{i j}\right\|=\left\|a_{i j}+d_{i j}\right\| \leq\left\|a_{i j}\right\|+\left\|d_{i j}\right\|
$$

to get upper bounds for $\left\|c_{i j}\right\|$ and $\left\|c_{j i}\right\|$ for use in the sufficient stability limits derived in Section 5 in the main text. 